\title{
Single-step purification of the recombinant green fluorescent protein from intact Escherichia coli cells using preparative PAGE
}

\begin{abstract}
Mechanical and non-mechanical breakages of bacterial cells are usually the preliminary steps in intracellular protein purification. In this study, the recombinant green fluorescent protein (GFP) was purified from intact Escherichia coli cells using preparative PAGE. In this purification process, cells disruption step is not needed. The cellular content of E. coli was drifted out electrically from cells and the negatively charged GFP was further electroeluted from polyacrylamide gel column. SEM investigation of the electrophoresed cells revealed substantial structural damage at the cellular level. This integrated purification technique has successfully recovered the intracellular GFP with a yield of $82 \%$ and purity of $95 \%$.
\end{abstract}

Keyword: Escherichia coli; Green fluorescent protein; Preparative PAGE; Protein purification 